\title{
Ritmicidad circadiana y adicción
}

\section{Circadian rhythmicity and addiction}

| Ana Adan

\section{RESUMEN}

El estudio de los aspectos cronobiológicos relacionados con la adicción es un campo de reciente desarrollo que ha recabado datos tanto básicos como clínicos de interés. El consumo de drogas afecta negativamente la expresión rítmica circadiana, produciendo un aplanamiento de las funciones y un retraso de los valores máximos, pudiendo incluso desembocar en un estado de desincronización del control endógeno. Además, los genes reloj Clock y Per2 se han mostrado asociados con la vulnerabilidad a la adicción, el primero regulando directamente la actividad dopaminérgica en el sistema de la recompensa y el segundo participando en la sensibilidad y abstinencia a las drogas. La tipología circadiana vespertina también se ha perfilado como una diferencia individual a tener en cuenta como factor de riesgo en el desarrollo de adicción a drogas. Finalmente, la reorganización de la ritmicidad circadiana con hábitos horarios regulares ajustados al ciclo luzoscuridad, la exposición a luz natural o artificial y la administración de melatonina pueden ser estrategias a utilizar en el tratamiento de las drogodependencias.

Palabras clave: cronobiologia; ritmicidad circadiana; genes reloj; tipología circadiana; melatonina; luminoterapia.
Departamento de Psiquiatría y Psicobiología Clínica. Facultad de

Psicología. Universidad de Barcelona.

Enviar correspondencia a:

Ana Adan. Dept. Psiquiatría y Psicobiología Clínica.

Facultad de Psicología. Universidad de Barcelona.

Passeig Vall d'Hebrón 171. 08035 Barcelona.

E-mail: aadan@ub.edu.

\section{ABSTRACT}

The chronobiological aspects of addiction constitute a recently developed field of study that has obtained interesting data from both ibasic and clinical research. Drug consumption has a negative effect on the expression of circadian rhythmicity, since it produces a flattening of the functions and a delay of maximal values, and can even cause a state of desynchronization of endogenous control. Moreover, it has been shown that the clock-genes Clock and Per2 are associated with vulnerability to addiction, the first gene directly regulating dopaminergic activity in the reward system and the second participating in the sensitivity to and abstinence from drugs. The evening circadian typology has also been proposed as an individual difference to be taken into account as a risk factor in the development of drug addiction. Finally, the reorganization of circadian rhythmicity with regular daily schedules adjusted to the cycle of light-darkness, exposure to natural or artificial light, and the administration of melatonin may be useful strategies in the treatment of drug addictions.

Key words: chronobiology; circadian rhythmicity; clock genes; circadian typology; melatonin; bright light. 
$\mathrm{L}$ a cronobiología, ciencia encargada de estudiar los fenómenos rítmicos, se halla en una fase de expansión creciente en la que no sólo se recaban datos de tipo básico sino hallazgos de indudable interés aplicado que se están ya utilizando tanto en abordajes terapéuticos como preventivos en muy diversos ámbitos. Cardiología y oncología son sólo dos ejemplos de especialidades médicas que en la actualidad se benefician de los conocimientos aportados por la cronobiología. Si bien el interés por el estudio de las relaciones entre la expresión rítmica y el consumo de sustancias psicoactivas es más reciente y se halla en fase menos avanzada de desarrollo, los conocimientos existentes en la actualidad alertan de su importancia y el objetivo del presente trabajo es exponer aquellos más relevantes y de interés para los profesionales de las drogodependencias.

\section{LA RITMICIDAD CIRCADIANA INDICADOR DE SALUD}

Existe una multiplicidad de ritmos biológicos, aunque los más estudiados tanto por su interés en relación al rendimiento socio-laboral como en la clínica son los circadianos o de duración de $24 \mathrm{~h}$. Éstos se producen endógenamente en el reloj biológico del organismo, el núcleo supraquiasmático del hipotálamo. En condiciones normales la expresión ritmica circadiana se halla sincronizada a la información ritmica ambiental, de entre la que destaca el ciclo solar de luz-oscuridad (Herzog, 2007; Levi y Schibler, 2007). El reloj endógeno controla la expresión rítmica de nuestro organismo con una extrema precisión a la vez que muestra una evidente capacidad de plasticidad para ajustar su fase a cambios ambientales, como por ejemplo la realización de un viaje transmeridiano el cual de forma brusca nos traslada a otra zona horaria.

La mayoría de parámetros tanto biológicos como comportamentales presenta una ritmicidad circadiana, si bien la temperatura corporal interna y la melatonina pueden destacarse como marcadores rítmicos ampliamente estudiados y con importantes implicaciones clínicas. La temperatura interna, con una función sinusoidal cuyo máximo se produce entre 17-20 h. y el mínimo entre 3-5 h. de la madrugada, se considera un estimador de la actividad cerebral y es bien conocida su desorganización en diversos trastornos del humor entre los que destaca la depresión endógena. La hormona melatonina, producida por la glándula pineal y considerada la señal de oscuridad del organismo, presenta una secreción con un marcado pico nocturno dependiente de la duración del fotoperiodo ambiental y valores mínimos durante la vigilia (Adan, 2009). La afectación ritmica puede producirse de diversas maneras, aunque la más frecuente consiste en el aplanamiento de la función. Esto es, por valores máximos inferiores y mínimos superiores. Así, en el caso de la temperatura ello supone una menor activación durante la vigilia y una menor capacidad de conciliar y mantener el sueño nocturno. La pérdida del pico de melatonina resulta en un claro detrimento de la cantidad y calidad del sueño, el cual puede incluso desorganizarse, a la vez que se disminuye la actividad antioxidante endógena del organismo y la actividad inmunológica (Reiter, 2007; Tekbas, Ogur, Korkmaz, Kilic y Reiter, 2008). La adecuada expresión ritmica es un indicador de salud y calidad de vida en todas las fases del ciclo vital.

\section{EL CONSUMO DE SUSTANCIAS PSICOACTIVAS AFECTA LA RITMICIDAD CIRCADIANA}

Son numerosos los estudios que han constatado que el consumo crónico de sustancias psicoactivas afecta negativamente la expresión rítmica circadiana. Éste produce un aplanamiento de las funciones y un retraso de los valores máximos, pudiendo incluso desembocar en un estado de desincronización del control endógeno(Perreau-Lenz y Spanagel, 2008; Perreau-Lenz, Zghoul, Rodríguez de Fonseca, Spanagel y Bilbao, 2009). Las sustancias con más estudios publicados son el alcohol y el tabaco, si bien cada vez parece más plausible que los datos puedan extrapolarse a la mayoría de drogas. El consumo crónico de alcohol disminuye la amplitud rítmica tanto de la temperatura corporal como de la melatonina, observándose una maximización de ello durante el sindrome de abstinencia. Asi, se ha observado la producción de hipotermia durante el vigilia y de hipertermia en las horas nocturnas junto a una considerable disminución de la secreción de melatonina nocturna y valores incrementados diurnos (Danel y Touitou, 2004, para una revisión). La afectación en el ritmo circadiano de melatonina se constata también en individuos jóvenes y sanos con una administración puntual vespertina de alcohol a dosis moderada (Rupp, Acebo y Carskadon, 2007). Este dato adquiere una especial relevancia y alerta sobre la necesidad de llevar a cabo estudios con el patrón de consumo intensivo intermitente de alcohol en adolescentes y jóvenes (Cadaveira, 2009), para delimitar tanto el posible impacto sobre la ritmicidad circadiana como la duración de éste.

La afectación de la expresión rítmica circadiana también se ha evidenciado en sujetos fumadores jóvenes y sanos. Los fumadores presentan una menor amplitud y un retraso horario del máximo o acrofase en los valores diurnos de activación subjetiva y estado afectivo respecto a los no fumadores, siendo ello especialmente relevante en aquellos con dependencia elevada (Adan y Sáchez-Turet, 2000; Adan, Prat y Sánchez-Turet, 2004). De forma similar, las variaciones diurnas de parámetros cardiovasculares presentan un retraso de fase y valores superiores en la segunda mitad del día en fumadores respecto a no fumadores (Adan y Sánchez-Turet, 1995) de mayor magnitud si la dependencia es elevada (Adan, Sánchez-Turet y Artés, 1998). La afectación rítmica cardiovascular en las fases iniciales de consumo puede resultar un estimador de riesgo para el desarrollo de patologías agudas y crónicas asociadas al tabaquismo. 


\section{GENES RELOJ Y VULNERABILIDAD A LA ADICCIÓN}

Polomorfismos en los genes reloj se han asociado recientemente a una variedad de trastornos psicopatológicos, incluida la adicción a drogas (Herzog, 2007; McClung, 2007). Un hallazgo que cabe destacar es el que ha relacionado la actividad del sistema de la recompensa con genes reloj, en concreto con el gen Clock, responsable de activar diversos genes circadianos (McClung, Sidiripoulo, Vitaterna, Takahashi y White, 2005). El estudio se realizó con ratones portadores de un gen Clock mutante, quienes se mostraban mucho más activos tanto previa administración de cocaína como tras su administración respecto a los controles. Así mismo, la respuesta del sistema de recompensa a la droga fue muy superior observándose una mayor elevación de los niveles de dopamina y de su enzima sintetizador. Los resultados señalan al gen Clock como un regulador directo de la actividad dopaminérgica en las áreas cerebrales de la recompensa.

El gen circadiano Period 2 (Per2), se ha mostrado esencial para inhibir la sensibilidad a los efectos tanto de la cocaína (Abarca, Albrecht y Spanagel, 2002) como del alcohol (Perreau-Lenz, et al., 2009). Los animales manipulados genéticamente con ausencia de la expresión de dicho gen presentan una respuesta incrementada a los efectos de ambas drogas y la pérdida de la conocida variación diurna de la sensibilidad. Recientemente, también se han constatado variaciones en el gen Per2 en humanos asociadas a consumo elevado de alcohol (Spanagel, 2009). Además, el consumo de drogas puede influir en la expresión génica y modificar la de los genes reloj estableciendo cambios funcionales permanentes asociados a la ingesta de distinta magnitud según la vulnerabilidad del individuo.

Si bien hemos comentado los trabajos genéticos con cocaina y alcohol, todo apunta a la posibilidad de generalizar los hallazgos a la totalidad de drogas de abuso (Perreau-Lenz y Spanagel, 2008; Falcon y McClung, 2009). La investigación en esta línea se halla básicamente en fase preclínica y aunque queda mucho camino por recorrer, puede que en un futuro estos conocimientos reviertan en abordajes terapéuticos más específicos a los disponibles en la actualidad para el tratamiento de las adicciones.

\section{LA TIPOLOGÍA CIRCADIANA VESPERTINA, FACTOR DE RIESGO}

No todos los individuos tienen idéntica expresión rítmica circadiana. La tipología circadiana es una diferencia individual sobre la que en las últimas dos décadas se está recabando abundante información. Los individuos se clasifican en tres tipologías circadianas (matutinos, intermedios o ningún tipo y vespertinos) a partir de sus autoevaluaciones a cuestionarios específicos de los que disponemos de versiones validadas para su utilización en población española (Caci, Deschaux, Adan y Natale, 2009). Los matutinos se acuestan y levantan temprano y sitúan su mejor momento para desa- rrollar actividades físicas y mentales a primeras horas del dia, en cambio los vespertinos se levantan y acuestan tarde manifestando sus óptimos a finales del día e incluso a primeras horas de la noche. Los desfases en las funciones rítmicas circadianas de los grupos extremos oscilan entre las 2 y las 12h., dependiendo del parámetro. El grupo ningún tipo suele presentar una posición intermedia y a él pertenece alrededor del $60 \%$ de la población adulta joven, mientras que los grupos extremos suponen un 20\% respectivamente. Matutinos y vespertinos no sólo difieren en la expresión rítmica, sino en rasgos de personalidad y hábitos, pudiéndose hablar de distintos estilos de vida (Adan, Natale y Caci, 2008 para una revisión).

El estudio pionero de Adan (1994) observó diferencias en el consumo de sustancias psicoactivas legales (nicotina, alcohol y cafeína) entre tipologías circadianas, siendo los sujetos vespertinos quienes consumen más de todas ellas. Trabajos posteriores desarrollados en diversos paises han confirmado la observación, siendo ello de especial relevancia en población adolescente (Andershed, 2005; Gau, Shang, Merikangas, Chiu, Soong y Cheng, 2007). Mientras que la tipología circadiana matutina parece ser un factor protector en el inicio y mantenimiento de consumo de drogas, la tipología vespertina puede considerarse un factor de riesgo. El vespertino inicia el consumo con estimulantes quizás para ajustar su grado de activación diurna a las demandas socio-ambientales cuando su estado se halla deficitario y el de depresores a finales del día cuando su nivel de activación excede el requerido en esos momentos. Así, los individuos vespertinos obtienen un refuerzo superior, lo que promueve el mantenimiento de la conducta (Adan y Sánchez-Turet, 2000; Adan et al., 2008). Si el consumo se convierte en habitual, se producirá la bien conocida espiral de estrés (Koob, 2006) aunque su magnitud sea quizás mayor en el vespertino extremo.

Una tipología vespertina extrema también supone un factor de riesgo en el desarrollo otros trastornos psicopatológicos, destacando los del humor (Natale, Adan y Scapellato, 2005; Grandin, Alloy y Abramson, 2006; McClung, 2007). Bajo esta perspectiva incluso se ha sugerido que el inicio del consumo de sustancias psicoactivas podría suponer en algunos de ellos una terapéutica de automedicación. Sea como sea, los cambios rítmicos que producirá el consumo todavia pueden agravar la presencia sintomatológica promocionando el incremento de la dosis consumida. Aunque son muchos otros los elementos que también participan en el inicio y mantenimiento del consumo de drogas, la tipología circadiana es uno de ellos. Los abordajes preventivos de las drogodependencias deberian incorporar la promoción de hábitos que beneficien la organización rítmica circadiana con especial énfasis en encauzar las pautas horarias de individuos con patrón vespertino extremo, mucho más frecuente durante la adolescencia (Andershed, 2005; Adan et al., 2008). 


\section{ESTRATEGIAS TERAPÉUTICAS CRONOBIOLÓGICAS}

Las estrategias encaminadas a reorganizar la expresión rítmica circadiana durante la desintoxicación y deshabituación suponen una excelente medida de ayuda para los pacientes. Ello es bien conocido por los profesionales, que con independencia del enfoque terapéutico pautan unos horarios estables del sueño-vigilia, de las comidas y de las actividades diarias que se desarrollen. Asi mismo, estos horarios se estipulan muy encarrilados con el ciclo solar luzoscuridad, esto es, con una fase avanzada o equiparable a la de la tipología circadiana matutina. Mantener estos hábitos tras el alta clínica es no sólo aconsejable sino muy recomendable para mejorar la calidad de vida y evitar recaídas, si bien se requieren trabajos longitudinales que exploren y cuantifiquen de forma objetiva tal extremo.

No siempre las simples medidas de control sobre hábitos horarios pueden ser suficientes en la recuperación de una ritmicidad dentro de valores homeostáticos 0 asociados a la salud. Es muy conveniente además cuidar que los pacientes se expongan a la luz diurna natural, lo que en nuestro país puede realizarse con enorme facilidad durante todo el año. Entre media hora o una hora de luz diaria (no es necesaria la presencia de sol), preferiblemente durante las horas matutinas, supone una terapia con potente efecto agonista melatoninérgico y serotoninérgico. Ello promociona que la pineal produzca un adecuado pico de melatonina nocturna para un sueño de mejor calidad (Barion y Zee, 2007) y reduce tanto el riesgo de padecer trastornos del humor como la presencia de sintomatología disfórica tan frecuente en las fases de abstinencia de drogas (Adan y Prat, 2007; Levi y Schibler, 2007). Cuando la exposición a la luz natural no es posible o resulta insuficiente, puede utilizarse la luminoterapia (http://www. yanche.com). Ésta consiste en la exposición a luz artificial de espectro total a intensidad de 10.000 lux, con una recomendación estándar de media hora al día de terapia. Exceptuando la existencia de retinopatías graves, la aplicación de luz artificial es una tratamiento muy seguro.

La administración de melatonina exógena resulta una posibilidad terapéutica cronofarmacológica (Reiter, 2007) a considerar en el futuro en el campo de las drogodependencias. La Agencia Europea de Evaluación del Medicamento autorizó su utilización en 2007, hallándose a punto de comercializarse en España a dosis de $2 \mathrm{mg}$. de liberación sostenida (Circadin ${ }^{\circledR}$ ). Tiene efecto organizador de la ritmicidad circadiana (hipnótico y activador diurno), por lo que supone un tratamiento más conservador y de primera opción respecto a los hipnóticos benzodiacepínicos y no benzodiacepínicos tradicionalmente usados. Entre sus cualidades cabe destacar que no es adictiva, no produce tolerancia y presenta un excelente perfil de seguridad, lo que le confiere un valor añadido para tratamientos a largo plazo y en poblaciones especiales (Adan y Prat, 2007; Adan, 2009). Su potente actividad antioxidante aporta un beneficio adicional tanto durante el tratamiento de desintoxicación y deshabituación como en pacientes dependientes que no cesan el consumo para minimizar el riesgo de neurodegeneración y la aparición de patología neuropsiquiátrica (Carvalho, 2009). En pacien- tes jóvenes y en especial en adolescentes el tratamiento debe establecerse de corta duración, siendo aconsejable efectuar analíticas para confirmar que existe un descenso de la producción endógena de melatonina. Para aquellos con más de 40 años puede optarse por la indicación de melatonina sin necesidad de determinaciones previas.

Pautar hábitos horarios, la exposición a la luz natural o artificial y la prescripción de melatonina, según el caso, con la finalidad de reorganizar la expresión rítmica circadiana son estrategias que pueden resultar muy beneficiosas en el manejo clínico de los pacientes drogodependientes. Éstas pueden mejorar la respuesta a los tratamientos, evitando la necesidad de prescribir determinados psicofármacos cuyo manejo en estos pacientes resulta más complejo por contraindicaciones y mayor riesgo de reacciones adversas o desarrollo de dependencia. Por último, su excelente seguridad permite utilizarlas durante períodos largos de tiempo e incluso de por vida, pudiendo resultar una medida protectora de recaídas o de aparición de sintomatología neuropsiquiátrica.

\section{AGRADECIMIENTOS}

Este trabajo ha contado con una subvención por parte del Ministerio de Ciencia e Innovación Español (proyecto PSI2009-12300, subprograma PSIC) y con el soporte del Departamento de Innovación, Universidades y Empresa de la Generalitat de Catalunya.

\section{REFERENCIAS}

Abarca, C., Albrecht, U. y Spanagel, R. (2002). Cocaine sensitization and reward are under the influence of circadian genes and rhythm. Proceedings of the National Academy of Sciences, 99, 90269030.

Adan, A. (1994). Chronotype and personality factors in the daily consumption of alcohol and psychostimulants. Addiction, 89, 455-462.

Adan, A. (2009). Melatonina: utilidad clinica en el anciano. Informaciones Psiquiátricas, 195-196, 21-32.

Adan, A., Natale, V. y Caci, H. (2008). Cognitive strategies and circadian typology. En: Léglise, A. L. (Ed.). Progress in circadian rhythms research. Pp. 141-161. Nova Biomedical Books. New York: Nova Science Publishers, Inc.

Adan, A. y Prat, G. (2007). Psicofarmacología en esquemas. Colección Salud. Barcelona: Publicacions i Edicions Universitat de Barcelona.

Adan, A., Prat, G. y Sánchez-Turet, M. (2004). Effects of nicotine dependence on diurnal variations of subjective activation and mood. Addiction, 98, 1599-1607.

Adan, A. y Sánchez-Turet, M. (1995). Smoking effects on diurnal variations of cardiovascular parameters. International Journal of Psychophysiology, 20, 189-198. 
Adan, A. y Sánchez-Turet, M. (2000). Effects of smoking on diurnal variations of subjective activation and mood. Human Psychopharmacology Clinical and Experimental, 15, 287-293.

Adan, A., Sánchez-Turet, M. y Artés, M. (1998). Interés clínico de las variaciones diurnas cardiovasculares en relación al consumo de tabaco y su grado de dependencia. Adicciones, 10, 43-51.

Andershed, A-K. (2005). In sync with adolescence: the role of morningness-eveningness in adolescence. New York: Springer.

Barion, A. y Zee, P. C. (2007). A clinical approach to circadian rhythm sleep disorders. Sleep Medicine, 8, 566-577.

Caci, H., Deschaux, O., Adan, A. y Natale, V. (2009). Comparing three morningness scales: age and gender effects, structure and cutoff criteria. Sleep Medicine, 10, 240-245.

Cadaveira, F. (2009). Alcohol y cerebro adolescente. Adicciones, 21, 9-14.

Carvalho, F. (2009). ¿Existe envejecimiento precoz entre los consumidores de drogas de abuso?. Adicciones, 21, 99-104.

Danel, T. y Touitou, Y. (2004). Chronobiology of alcohol: from chronokinetics to alcohol-related alterations of the circadian system. Chronobiology International, 21, 923-935.

Falcon, E. y McClung, C. A. (2009). A role for the circadian genes in drug addiction. Neuropharmacology, 56, 91-96.

Gau, S. S., Shang, C. Y., Merikangas, K. R., Chiu, Y. N., Soong, W. T. y Cheng, A. T. (2007). Association between morningnesseveningness and behavioral/emotional problems among adolescents. Journal of Biological Rhythms, 22, 268-274.

Grandin, L. D., Alloy, L. By Abramson L. Y. (2006). The social zeitgeber theory, circadian rhythms, and mood disorders: review and evaluation. Clinical Psychology Review, 26, 679-694.

Herzog, E. D. (2007). Neurons and networks in daily rhythms. Nature Reviews, 8, 790-802.
Levi, F. y Schibler, U. (2007). Circadian rhythms: mechanisms and therapeutic implications. Annual Review of Pharmacology and Toxicology, 47, 593-628.

McClung, C. A. (2007). Circadian genes, rhythms and the biology of mood disorders. Pharmacology \& Therapeutics, 11, 222-232.

McClung, C. A., Sidiripoulo, K., Vitaterna, M., Takahashi, J. S. y White, F. J. (2005). Regulation of dopaminergic transmission and cocaine reward by the Clock gene. Proceedings of the National Academy of Sciences, 102, 9377-9381.

Natale, V., Adan, A. y Scapellato, P. (2005). Are seasonality of mood and eveningness closely associated? Psychiatry Research, 136, 51-60.

Perreau-Lenz, S. y Spanagel, R. (2008). The effects of drugs of abuse on clock genes. Drug News \& Perspectives, 21, 211-217.

Perreau-Lenz, S., Zghoul, T., Rodríguez de Fonseca, F., Spanagel, R. y Bilbao, A. (2009). Circadian regulation of central ethanol sensitivity by the mPer2 gene. Addiction Biology, 14, 253-259.

Reiter, R. J. (2007). Medical implications of melatonin: receptormediated and receptor-independent actions. Advances in Medical Sciences, 52, 11-28.

Rupp, T. L., Acebo, C. y Carskadon, M. A. (2007). Evening alcohol suppresses salivary melatonin in young adults. Chronobiology International, 24, 463-470.

Spanagel, R. (2009). Alcoholism: a systems approach from molecular physiology to addictive behaviour. Physiological Reviews, 89, 649-705.

Tekbas, O. F., Ogur, R., Korkmaz, A., Kilic, A. y Reiter, R. J. (2008). Melatonin as an antibiotic: new insights into the actions of this ubiquitous molecule. Journal of Pineal Research, 44, 222-226. 
